\title{
Abuse among elderly living in old age homes of Pokhara Lekhnath Metropolitan City
}

\author{
Mira Adhikari Baral, Pramila Baral, and Bhagwati K.C
}

This article has been withdrawn by bioRxiv as it is not within scope and posted owing to a screening error. 